\title{
P02-77
}

\section{CAN SALIVARY CORTISOL ASSESSMENT FROM A SINGLE HF-RTMS SESSION PREDICT CLINICAL OUTCOME IN MELANCHOLIC DEPRESSED PATIENTS? A PILOT STUDY}

C. Baeken ${ }^{1}$, L. Santermans ${ }^{1}$, D. Zeeuws ${ }^{1}$, R. De Raedt ${ }^{2}$, N. Vanderbruggen ${ }^{1}$

${ }^{1}$ Psychiatry, UZBrussel, Brussel, ${ }^{2}$ Psychology, UGent, Ghent, Belgium

Aim: The underlying physiological mechanisms as to why repetitive transcranial magnetic stimulation (rTMS) can treat depressed patients remains unclear and no clear biological markers are available to indicate a positive outcome for a given patient. Some data suggest that rTMS can influence hypothalamic-pituitary-adrenal (HPA) processes. In this study, we wanted to evaluate whether one session of high frequency (HF)-rTMS applied on the left dorsolateral prefrontal cortex (DLPFC) can predict a successful treatment outcome in a sample of antidepressant free unipolar depressed patients of the melancholic subtype.

Methods: Twenty one right-handed treatment resistant depressed patients were studied. Fifty two percent of the patients were considered as treatment responders, as defined by a $50 \%$ reduction in her/his baseline 17 -item Hamilton Depression Rating Scale (HDRS) score. To examine acute HF-rTMS effects on the HPA- axis, we analyzed salivary cortisol levels, using a sham-controlled, 'single' blind, crossover design. Areas under the curves (AUC) were calculated for both real and sham HF-rTMS.

Results: Real HF-rTMS resulted in salivary cortisol decreases, however only in HF-rTMS non-responders. No HF-rTMS influences were found in the responder group. Sham stimulation did not affect the HPA-axis. Non-responders were found to display a higher level of co-morbid personality disorders.

Conclusion: Our results may provide more insight into the underlying working mechanisms of HF-rTMS and could add further information about endocrinological functioning in affective disorders. Furthermore, personality features in combination with a 'supersensitive' HPA system could be of importance to predict clinical outcome. 